\title{
EDITORIAL
}

\section{A novel conceptual framework for psychiatry: vertically and horizontally integrated approaches to redundancy and pleiotropism that co-exist with a classification of symptom clusters based on DSM-5}

Molecular Psychiatry (2013) 18, 846-848; doi:10.1038/mp.2013.90

Since the release of the fifth edition of the Diagnostic and Statistical Manual (DSM-5) of the American Psychiatric Association, ${ }^{1}$ there has been a flurry of stories in the popular press, as well as in scientific journals, ${ }^{2-11}$ about this new version of psychiatric nosology. There are some differences from its preceding edition, such as the exclusion of Asperger syndrome as a separate diagnosis (subsumed now in the autism spectrum), loss of subtype classifications for variant forms of schizophrenia, dropping the 'bereavement exclusion' for depressive disorders and a new category of gambling disorder. Within the large universe of psychiatric disorders, these represent few changes, albeit fraught with repercussions for those affected by them, such as the increasingly large community that refers to themselves as 'Aspies,' identifying with a diagnosis of Asperger's that no longer exists. What we find most remarkable in this immense debate is the intense level of passion.

One of us (JL) recently reviewed a book for Science, entitled The Book of Woe: The DSM and the Unmaking of Psychiatry by Gary Greenberg. ${ }^{12,13}$ A reading of Greenberg's book and related materials revealed how passionate and partisan stakeholders are on this matter. It seems that those who have a grudge against psychiatry are using the opportunity of its launch to criticize not only the DSM-5 itself, but also the entire field of psychiatry, making the illogical statement that if we cannot classify psychiatric disorders with absolute precision, then the disorders do not exist and consequently the entire field is nothing but a hoax, populated exclusively by self-serving villains.

The simple-mindedness and lack of internal consistency of these passionately venomous critics is astounding. Although the DSM-5 is a symptomatic classification system, based on clusters of clinical presentations, causes exist in the realms of biology, psychology and society, with the major complications of redundancy and pleiotropism. In other words, we have known disorders of the mind since antiquity by their symptomatic presentations, which, in a refined format, are the underlying basis of the DSM-5. Therefore, one is classified as depressed, schizophrenic or autistic based on the constellation of symptoms one presents with. There is nothing intrinsically wrong in grouping clusters of symptoms in this manner. DSM- 5 makes no claim that each of its categories represent a distinct biological entity with uniquely defined pathogenesis. Things start to go awry when we make efforts to do just that. Such efforts have failed because of the phenomena of redundancy and pleiotropism, which in our view underlie most, if not all, causation mechanisms in psychiatry. We use these terms here with slight variations of their common use. Therefore, to avoid confusion, let us clarify these two concepts, as we apply them to psychiatry.

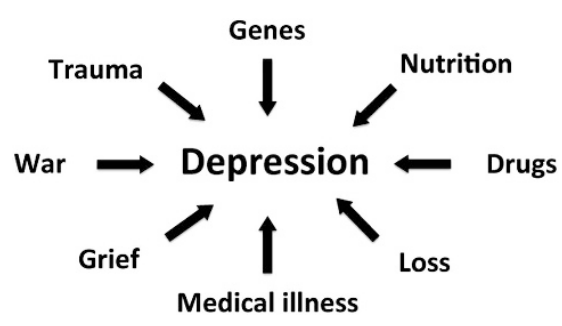

Figure 1. An example of redundancy in psychiatry. Several diverse causal or contributory factors can act in isolation or in different types of combination to result in similar clusters of symptoms.

\section{REDUNDANCY}

Redundancy has multiple meanings, including those of duplication, excess and repetition. Symptomatic clusters, also known as psychiatric diagnoses, can each be the result of multiple causes. Figure 1 shows in a simplified manner, which was first proposed by us in 2001,14 that similar clinical presentations of depressive disorders can be caused by a variety of factors, ranging from the biological, to the psychological and social, alone or in various combinations. It would be logical to assume that the more such risk factors one accumulates, the more one is likely to have major depression. In an outstanding Molecular Psychiatry article Kendler and Halberstadt ${ }^{15}$ have recently shown that monozygotic twins can be discordant for major depression in the context of different life trajectories. Therefore, it is not possible to pinpoint any single factor as being by itself necessary and sufficient to cause a psychiatric disorder. Most likely, the affected patients have multiple risk factors in different combinations that vary individually from patient to patient.

\section{PLEIOTROPISM}

Pleiotropism refers the concept of one single factor having multiple effects. The concept of genetic pleiotropism implies that a single gene can cause different phenotypes, depending on various interactions at the gene-gene and gene-environmental levels. As such interactions can vary drastically across individuals, the manifestations of a single genetic change can likewise be highly diverse. A prime example is Marfan syndrome, known to be caused by mutations in the FBN1 gene on chromosome 15, which encodes the glycoprotein fibrillin-1, a component of the extracellular matrix. ${ }^{16}$ Clinically, the diagnosis is made through the revised Ghent nosology for the Marfan syndrome. ${ }^{17}$ This consists of a collection of clinical features; each assigned a score, as follows:

- Wrist and thumb sign-three (wrist or thumb sign-1)

- Pectus carinatum deformity-two (pectus excavatum or chest asymmetry-1)

- Hindfoot deformity-two (plain pes planus-1) 


\section{Depression

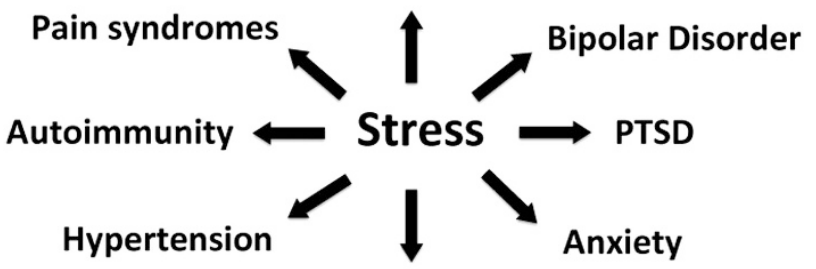 \\ Metabolic syndrome}

Figure 2. An example of pleiotropism in psychiatry. A single factor can be the cause of or contribute to a variety of diverse psychiatric and medical disorders.

- Pneumothorax-two

- Dural ectasia-two

- Protrusio acetabuli-two

- Reduced upper segment/lower segment and increased arm/ height and no severe scoliosis-1

- Scoliosis or thoracolumbar kyphosis-1

- Reduced elbow extension-1

- Facial features (3/5)-1 (dolichocephaly, enophthalmos, downslanting palpebral fissures, malar hypoplasia and retrognathia)

- Skin striae-1

- Myopia>3 diopters-1

- Mitral valve prolapse (all types)-1

The diagnosis of Marfan is made after a score threshold is reached. It is self-evident that any individual feature of Marfan listed above can be caused by the most varied conditions. The diagnosis of Marfan syndrome is given by their clinical clustering. Moreover, some individuals may meet the clinical cluster and not have the causative FBN1 gene mutations, whereas others with the mutations have no or few clinical features and may not meet clinical diagnostic criteria. This type of pleiotropism is most likely found in psychiatric disorders as well. For example, several papers published in recent issues of Molecular Psychiatric show that the same genetic variants can cause either bipolar disorder and major depression ${ }^{18}$ or bipolar disorder and schizophrenia. ${ }^{19-21}$ There is also the concept of pharmacological pleiotropism, according to which the same drug causes multiple, unrelated biological effects. If one looks at pathogenesis in psychiatry one can see abundant examples of such pleiotropism. For example, stress can cause or contribute to a variety of psychiatric and medical conditions, as shown in Figure 2.

\section{A CONCEPTUAL FRAMEWORK FOR PSYCHIATRY}

As various combinations of dissimilar causes can lead to the same cluster of symptoms (redundancy) and as the same causal or contributory factor can lead to the most diverse presentations (pleiotropism), we may never be able to equate clusters of symptoms with specific and distinct causes. This neither negates the existence of symptomatic clusters nor invalidates a clinical, presentation-based nosology, which can-and should-co-exist with our efforts to establish causation. We therefore propose a conceptual framework for psychiatry that is shown in Figure 3. In that model, we have at the level of the brain, known, defined changes (for example, the plaques and tangles of Alzheimer's disease) or yet to be discovered or confirmed microstructural or functional changes, leading to major and impairing symptoms that affect higher mental processes, such as mood, memory and cognition. A useful way to approach psychiatric disorders is to foster vertical translational integration, which includes research done at the levels of cells, animal models, first to human translation, clinical trials, new treatments and improved outcomes,
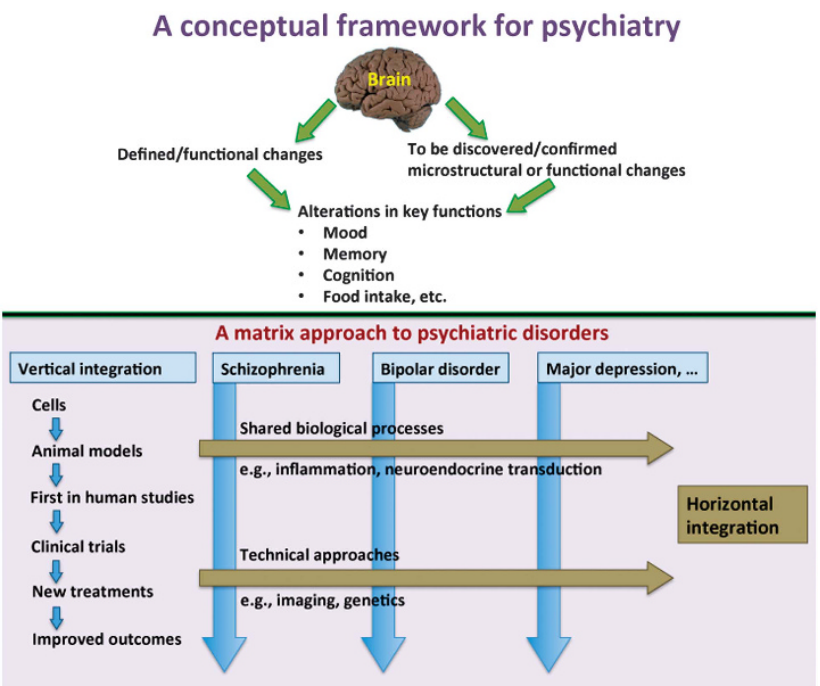

Figure 3. A conceptual framework for psychiatry. Top panel: in the brain defined structural changes, such as the plaques and tangles of Alzheimer's disease, or to be discovered or confirmed microstructural or functional changes can lead to key symptoms. Bottom panel: parallel and simultaneous tracks of integration along both thematic or technical horizontal axes and at the vertical translational level are needed in psychiatry.

in parallel with horizontal integration, at two levels: along biological pathways and processes, such as inflammation and neuroendocrine transduction, and along technical approaches, such as imaging or genetic sequencing.

Reductionistic thinking that tries to negate the existence and the resultant burden of psychiatric disorders is the unfortunate product of biased and misinformed minds. Insisting on nothing but a purely descriptive dissection of symptoms is likewise an obstinate effort that will not lead to advances in the field. Very intensive, rigorous, and meticulous collaborative efforts that lead to both vertical and horizontal integration of research efforts are at present the most likely pathway for substantial advances for psychiatry. The US National Institute of Mental Health (NIMH) has proposed Research Domain Criteria (RDoC), which are based on studying at a dimensional level the genetic, neural, and behavioral features of mental disorders, with an integrative approach that includes cognition along with social processes, arousal/regulatory systems, and negative- and positive-valence systems as the major domains relevant to psychiatry. ${ }^{22}$ This is an interesting attempt to go beyond current nosological approaches. It remains to be seen whether the RDoC will stand the test of time. Regardless of the success of this specific approach, in our opinion the best pathway forward will consist of investigating psychiatric disorders along both (translational) vertical and (thematic/technical) horizontal axes, as shown in the lower part of Figure 3, in conjunction with traditional, symptom-based approaches to nosology, such as DSM-5.

\section{CONFLICT OF INTEREST}

The authors declare no conflict of interest.

$\mathrm{J}$ Licinio and M-L Wong Mind and Brain Theme, South Australian Health and Medical Research Institute and Department of Psychiatry, School of Medicine, Flinders University Adelaide, SA, Australia

E-mail: julio.licinio@sahmri.com 


\section{REFERENCES}

1 American Psychiatric Association, and American Psychiatric Association DSM-5 Task Force. Diagnostic and Statistical Manual of Mental Disorders: DSM-5. American Psychiatric Association: Arlington, VA, USA, 2013.

2 Berk M. BMC Med 2013; 11: 128.

3 Buxbaum JD, Baron-Cohen S. Mol Autism 2013; 4: 11.

4 Carpenter WT, Tandon R. Asian J Psychiatr 2013; 6: 266-268.

5 Frances A. BMJ 2013; 346: f1580.

6 Halter MJ, Rolin-Kenny D, Dzurec LC. J Psychosoc Nurs Ment Health Serv 2013; 51: 30-39.

7 Malhi GS. Aust N Z J Psychiatry 2013; 47: 7-9.

8 Paris J. Making the DSM-5: CONCEPTS and Controversies. Springer: New York, NY, USA, 2013.

9 Ritvo ER, Ritvo RA. Am J Psychiatry 2013; 170: 444-445.

10 Smoyak SA, Halter MJ. J Psychosoc Nurs Ment Health Serv 2013; 51: 4-5.

11 Tandon R, Gaebel W, Barch DM, Bustillo J, Gur RE, Heckers S et al. Schizophr Res 2013 Jun 22. pii: S0920-9964(13)00283-1. doi:10.1016/j.schres.2013.05.028. (Epub ahead of print).
12 Greenberg G. The Book of Woe: The DSM and the Unmaking of Psychiatry. Blue Rider Press: Penguin: New York, NY, USA, 2013.

13 Licinio J. Science 2013; 340: 1406-1407.

14 Wong ML, Licinio J. Nature Rev Neurosci 2001; 2: 343-351.

15 Kendler KS, Halberstadt LJ. Mol Psychiatry 2012, doi:10.1038/mp.2012.55. (Epub ahead of print).

16 McKusick VA. Nature 1991; 352: 279-281.

17 Loeys BL, Dietz HC, Braverman AC, Callewaert BL, De Backer J, Devereux RB et al. Med Genet 2010; 47: 476-485.

18 Liu Y, Blackwood DH, Caesar S, de Geus EJ, Farmer A, Ferreira MA et al. Mol Psychiatry 2011; 16: 2-4.

19 Williams HJ, Norton N, Dwyer S, Moskvina V, Nikolov I, Carroll L et al. Mol Psychiatry 2011; 16: 429-441.

20 Dwyer S, Williams H, Jones I, Jones L, Walters J, Craddock N et al. Mol Psychiatry 2011; 16: 790-791.

21 Christoforou A, McGhee KA, Morris SW, Thomson PA, Anderson S, McLean A et al. Mol Psychiatry 2011; 16: 240-242.

22 Morris SE, Cuthbert BN. Dialogues Clin Neurosci 2012; 14: 29-37. 